Article

\title{
Drift of Scroll Waves in a Mathematical Model of a Heterogeneous Human Heart Left Ventricle
}

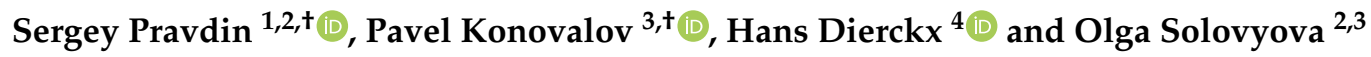 \\ and Alexander V. Panfilov 2,5,*(D) \\ 1 Krasovskii Institute of Mathematics and Mechanics, 620990 Ekaterinburg, Russia; sfpravdin@imm.uran.ru \\ 2 Laboratory of Computational Biology and Medicine, Ural Federal University, 620075 Ekaterinburg, Russia; \\ o.solovyova@iip.uran.ru \\ 3 Institute of Immunology and Physiology UB RAS, 620049 Ekaterinburg, Russia; p.konovalov@iip.uran.ru \\ 4 KULeuven Campus KULAK, 8500 Kortrijk, Belgium; h.dierckx@kuleuven.be \\ 5 Department of Physics and Astronomy, Ghent University, 9000 Ghent, Belgium \\ * Correspondence: Alexander.Panfilov@ugent.be \\ + These authors contributed equally to this work.
}

Received: 24 March 2020; Accepted: 6 May 2020; Published: 12 May 2020

\begin{abstract}
Rotating spiral waves of electrical excitation underlie many dangerous cardiac arrhythmias. The heterogeneity of myocardium is one of the factors that affects the dynamics of such waves. In this paper, we present results of our simulations for scroll wave dynamics in a heterogeneous model of the human left ventricle with analytical anatomically based representation of the geometry and anisotropy. We used a set of 18 coupled differential equations developed by ten Tusscher and Panfilov (TP06 model) which describes human ventricular cells based on their measured biophysical properties. We found that apicobasal heterogeneity dramatically changes the scroll wave dynamics. In the homogeneous model, the scroll wave annihilates at the base, but the moderate heterogeneity causes the wave to move to the apex and then continuously rotates around it. The rotation speed increased with the degree of the heterogeneity. However, for large heterogeneity, we observed formation of additional wavebreaks and the onset of complex spatio-temporal patterns. Transmural heterogeneity did not change the dynamics and decreased the lifetime of the scroll wave with an increase in heterogeneity. Results of our numerical experiments show that the apex may be a preferable location of the scroll wave, which may be important for development of clinical interventions.
\end{abstract}

Keywords: spiral wave; heterogeneity; heart modeling; myocardium; left ventricle

\section{Introduction}

Vortices in excitable medium, which are called spiral waves in 2D or scroll waves in 3D, were found in many physical, chemical, and biological systems [1]. They play an important role in dynamics of these systems. For example, formation of such vortices in the heart causes cardiac arrhythmias, which remain the largest cause of death in the industrialized countries [2]. The dynamics of such vortex in the heart determines the type of cardiac arrhythmia. For example, the drift of a scroll wave in the ventricles of the heart may result in the onset of an arrhythmia called polymorphic ventricular tachycardia [3] and a breakup of the vortex into complex spatio-temporal patterns results in the onset of ventricular fibrillation, which causes cardiac arrest and sudden cardiac death [4]. Therefore, the factors responsible for drift of scroll waves are of great interest.

In a very general sense, the factors which can cause drift of a scroll wave include geometrical factors, anisotropy, and tissue heterogeneity. Previous research on generic models showed that, in homogeneous isotropic medium with high excitability, scroll waves drift to the regions where the 
thickness of the domain is minimal $[5,6]$. This result was recently confirmed in studies using detailed models of cardiac tissue [7]. Anisotropy of cardiac tissue also causes drift of vortices in 2D [8] and 3D [9]. Another important factor is the tissue heterogeneity, which will be a main focus of this paper.

The human heart, as in other mammals, has two main types of normal heterogeneity: transmural [10-12] and apicobasal [13,14]. The transmural heterogeneity in the human left ventricle (LV) involves an increase in action potential duration (APD) from subendocardial to subepicardial regions [15]. At the same time, data on the apicobasal heterogeneity are controversial: some papers report longer APD [13,14] while other show shorter APD [16] at the LV apex in comparison with the LV base. In a normal heart, this heterogeneity minimizes the dispersion of repolarization of cardiomyocytes from different regions and, as a consequence, makes the heart contraction maximally effective [17]. However, in pathological conditions, the heterogeneity amplifies and plays an important role during cardiac arrhythmias [18,19].

The effect of heterogeneity on vortex dynamics has mainly been studied for 2D vortices, known as spiral waves. It was shown in generic [20] and in ionic models [21] of cardiac tissue that heterogeneity in APD causes drift of a spiral wave to the regions of longer period. The effect of transmural heterogeneity on scroll waves was studied only in generic models [22,23], and it was shown that small heterogeneity transversal to the plane of rotation of a scroll wave results in the onset of a twisted scroll wave, i.e., the spiral waves have different rotation phase in different sections [22]. However, a large heterogeneity can cause a breakup of a scroll wave into a complex spatio-temporal pattern [23].

Because a real heart has regions with different thickness, anisotropy, and heterogeneity, all of these factors can potentially affect dynamics of scroll waves during cardiac arrhythmia. Recently, studies of effects of some of these factors on scroll wave dynamics in a model human ventricle were performed. A three-dimensional model describes the shape and anisotropy of the left ventricle of the human heart using a small number of parameters and has been verified against histological and DT-MRI data [24]. Using this geometrical model, the effect of the thickness of myocardial wall and anisotropy of cardiac tissue $[7,25]$ has been studied. It was shown that the scroll wave usually stabilizes at a certain region of the ventricle; this location is mainly affected by the thickness of the wall and anisotropy.

Recently [26], we studied the effects of apicobasal heterogeneity using a two-variable model of cardiac tissue by Aliev and Panfilov [27]. This model belongs to a class of low-dimensional models of cardiac tissue which describe generic properties for waves propagating in the heart, but do not reproduce a detailed biophysical mechanism for cardiac excitation. We found that heterogeneity has some effect on the location of the scroll wave, but only if it was extremely large, 200 ms or more. Although such low-dimensional model reproduces the generic dynamics of waves in the heart [28], to obtain more qualitative estimates, one needs to use ionic models which describe the underlying mechanisms of action potential generation. Recently, one such study using a detailed ionic model for human ventricular cell TP06 was performed [29]. It was shown that the use of the ionic model substantially affected the observed dynamics of the vortices. In particular, it was found that, in most of the cases, we do not see any stabilization as we did in the AP model. On the contrary, the scroll wave drifts to the base of the heart and disappears. The main aim of this paper is to perform a study of scroll wave dynamics in a realistic ionic model of human ventricular tissue which includes not only correct shape and anisotropy, but also the heterogeneity of the heart ventricles.

\section{Materials and Methods}

\subsection{Baseline Homogeneous Model. Numerical Approach and Software}

The numerical computation of electrophysiological activity requires models at three levels: cell, tissue, and organ.

We used the LV anatomical model described in [24]. This model is axisymmetric and represents an average ventricle of a healthy adult human. The LV model was represented as a body of revolution with the shape fitted to experimental data [30]. The rotation axis is vertical axis $\mathrm{Oz}$. An example of the 
LV model is shown in Figure 1C. The epicardium is the colored surface, and the endocardium is the mesh surface.
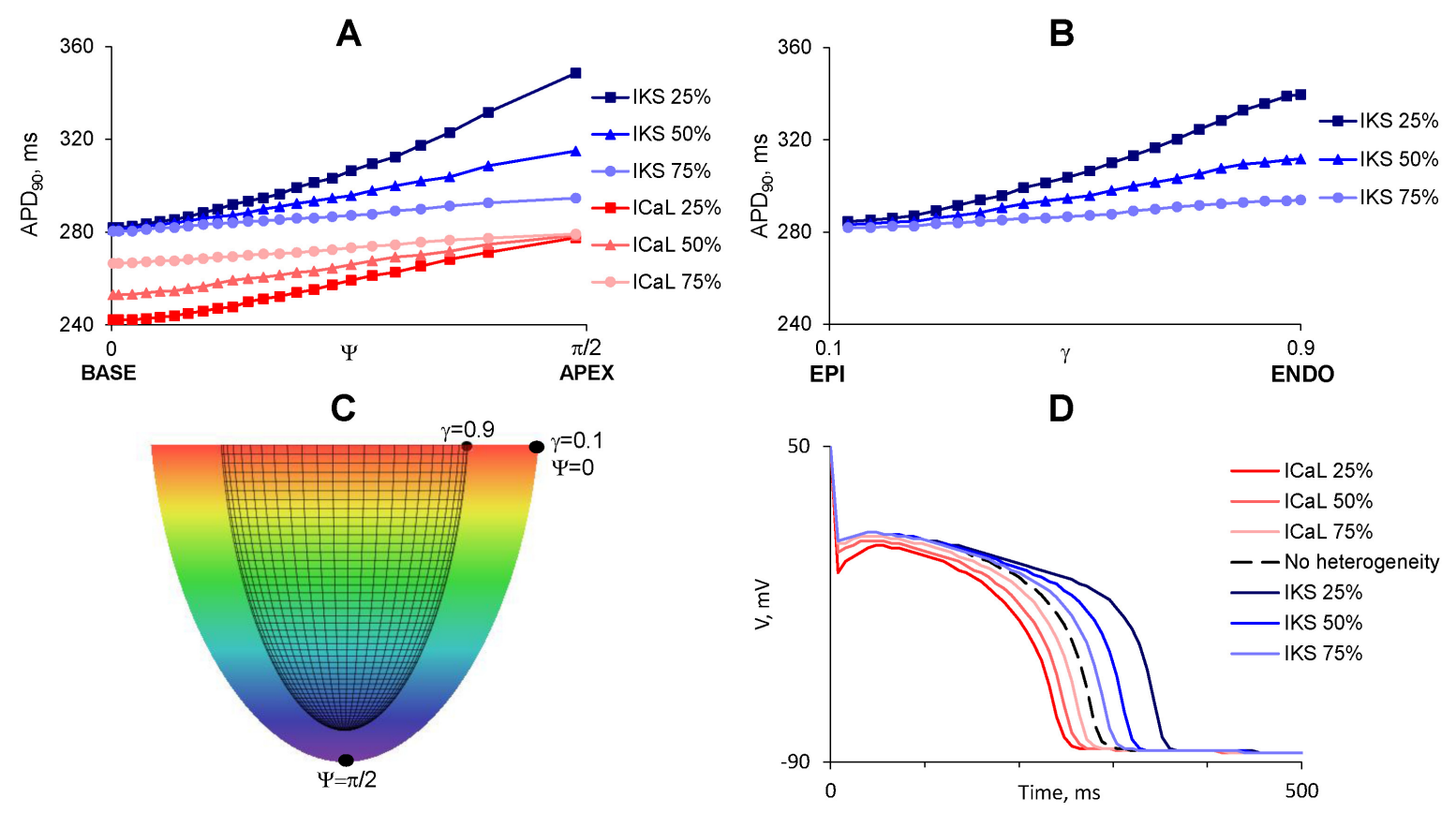

Figure 1. Distribution of $A P D_{90}$ (shown by color, from blue, $353 \mathrm{~ms}$, to red, $248 \mathrm{~ms}$ ) in the considered heterogeneous models of the LV. (A) apicobasal heterogeneity is set by a decrease of IKs at the apex (shades of blue) or ICaL at the base (shades of red). APD90 increases with increase of $\Psi$ from 0 (base) to $\pi / 2$ (apex); (B) Transmural heterogeneity is set by decrease of IKs at the subendocardium. $\gamma=0.1$ means subepicardium cells, $\gamma=0.9$, subendocardial; (C) points with maximal difference of APD90 in cases of apicobasal $(\Psi=0, \Psi=\pi / 2)$ and transmural $(\gamma=0.1, \gamma=0.9)$ heterogeneity. Color shows the $z$-coordinate; (D) plot of the action potential against time in LV points with maximal heterogeneity.

The model has the following list of parameters. $R_{b}$ is the LV outer radius on the LV equator; $Z_{b}$ is the LV height including the apical wall thickness; $L$ is the LV wall thickness at the LV equator; $h$ is the $\mathrm{LV}$ wall thickness at the LV apex; and $\epsilon \in[0,1]$ is a dimensionless parameter influencing the conicity-ellipticity characteristic of the LV shape.

A spherical-like local coordinate system $(\gamma, \psi, \phi)$ is connected with the LV model. Its coordinate $\gamma$ gives position in the wall from the endocardium $\gamma=\gamma_{0}$ to the epicardium $\gamma=\gamma_{1} ; \psi$ is an analogue of geographical longitude with the base, $\psi=0$, and the apex, $\psi=\pi / 2 ; \phi \in[0,2 \pi)$.

The local coordinates are linked with the cylindrical coordinates $(\rho, \varphi, z)$ by the following formulae:

$$
\begin{aligned}
& \rho=\left(R_{b}-\gamma L\right)(\epsilon \cos \psi+(1-\epsilon)(1-\sin \psi)), \\
& \varphi=\phi, \\
& z=\left(Z_{b}-\gamma h\right)(1-\sin \psi)+(1-\gamma) h .
\end{aligned}
$$

In every LV point, a vector of myofibre direction is defined as described in [24].

The parameters of the anatomical model were kept constant between all our simulations: outer (epicardial) radius at the base $R_{b}=33 \mathrm{~mm}$, basal thickness $L=12 \mathrm{~mm}$, full height $Z_{b}=60 \mathrm{~mm}$, and ellipticity coefficient $\varepsilon=0.85$.

A set of parameters was taken as reference. The reference ventricle had anisotropy coefficients in ratio 9:1, transmural fibre rotation angle (FRA) of $147^{\circ}$, and apical LV thickness $h=6 \mathrm{~mm}$. 
The LV model allows us to easily change LV geometrical parameters-for example, to vary its thickness at the base and apex. These parameters are important for studying scroll waves drift which goes, accordingly with scroll waves theory, to a thin place if filament tension is positive.

For the tissue level, we used a monodomain approach [31] and anisotropic medium. Reaction-diffusion equations describe processes in the modelled body and have the following form:

$$
\begin{gathered}
\frac{\partial u}{\partial t}=\operatorname{div}(D \operatorname{grad} u)-\frac{I_{\text {ion }}}{C_{m}}, \\
I_{i o n}=I_{K r}+I_{K s}+I_{K 1}+I_{t o}+I_{N a}+I_{b N a}+I_{C a L}+I_{b C a}+I_{N a K}+I_{N a C a}+I_{p C a}+I_{p K} .
\end{gathered}
$$

Here, $\mathrm{u}=\mathrm{u}(\mathrm{r}, \mathrm{t})$ is the transmembrane potential; the intracellular processes are captured by $I_{i o n}=I_{i o n}(r, t)$, which is the sum of the ionic transmembrane currents. $C_{m}$ is the capacitance of the cell membrane. In a general sense, this equation shows that the potential difference $V_{m}$ over the cell membrane changes due to currents $(I)$ which flow through the membrane. These currents are conveyed by different ions $(\mathrm{Na}, \mathrm{K}, \mathrm{Ca})$ and via various biophysical processes. In modern computational cardiac electrophysiology, the properties of all such currents are fitted to their experimentally measured values and their dynamics is fitted using additional differential equations. Each of the currents typically depends on $V_{m}$ and time, and the time-dependency is normally given by an exponential relaxation equation for the gating variable(s) $g$. For example, consider a hypothetical current $I_{*}$ which conveys ion ' $*$ ':

$$
\begin{gathered}
I_{*}=G_{*} g_{*}^{\alpha} g_{\bullet}^{\beta}\left(V_{m}-V_{*}\right) \\
\frac{d g_{i}}{d t}=\frac{g_{i}^{\infty}\left(V_{m}\right)-g_{i}}{\tau_{i}\left(V_{m}\right)}, i=*, \bullet,
\end{gathered}
$$

It has a maximal conductivity of $G_{*}=$ const and is zero for $V_{*}$, the so-called Nernst potential for ion ' $*$ '. This Nernst potential can be easily computed from the concentrations of specific ions outside and inside the cell membrane. The time dynamics of this current is given by two gating variables $g_{*}, g_{\bullet}$ to the power $\alpha, \beta$. The variables $g_{*}, g_{\bullet}$ approach their voltage-dependent steady state values $g_{i}^{\infty}\left(V_{m}\right)$ with characteristic time $\tau_{i}\left(V_{m}\right)(4)$. All parameters and functions are chosen here to fit experimentally measured properties of the specific ionic current. Most of ionic currents have one or two gating variables, with $\alpha=\beta=1$. In our simulations we use equations derived and fitted in [32], which has 18 state variables. Note that a proper description of ionic currents is very important as most cardiac drugs affect the maximal conductivities of specific ion channels. Furthermore, cardiac tissue is heterogeneous with respect to expression levels of these channels. Thus, using Equations (2)-(4), one can model the effect of pharmacological preparations on cardiac tissue, and, as we do in this paper, study effects of heterogeneity of the heart on cardiac arrhythmias. One of the standard ways to do so is to make the maximal conductivity of a specific current dependent on space in a manner based on experimental data. As described in the next subsection, the most important currents accounting for the heterogeneity are $I_{C a L}$ and $I_{K s}$.

As in [33], the diffusion matrix $D=\left(D^{i j}\right)$ was computed from the unit vectors in fibre direction $\mathbf{v}$ using the formula

$$
D^{i j}=D_{2} \delta_{i, j}+\left(D_{1} D_{2}\right) v_{i} v_{j},
$$

where $D_{1}$ and $D_{2}$ are the diffusion coefficients along and across the fibres and $\delta_{i, j}$ is the Kronecker symbol.

Ionic model parameters were taken from [32]; they correspond to physiological characteristics of cardiomyocytes.

A scroll wave was initiated using a standard protocol S1S2 [29] and rotated counterclockwise. We used a standard boundary condition - zero flux of potential through the LV surface. A uniform grid with spatial step $d r=0.28 \mathrm{~mm}$ was set in Cartesian coordinates. 
To solve the differential Equations (1)-(3), we used a finite difference approach. To approximate the diffusion term, we used a stencil of 18 points for 3D using the following equation:

$$
L(i, j, k)=\sum_{l=0}^{18} w_{l} V_{m}(l)
$$

where $l$ is an index over the 18 neighbors of the point $(i, j, k)$ including itself, and $w_{l}$ is the weight of the voltage of a particular neighboring grid point which accounts for its contribution to the Laplacian. The weights $w_{l}$ were computed based on the conduction tensor $D_{i j}$ and location of the point with respect to the local boundary. All points outside the heart geometry have weights $w_{l}=0$. The weights were precomputed for each geometry used and allowed an efficient evaluation of the Laplacian during the simulations. The gating variables in the TP06 model were integrated using the Rush and Larsen approach [34]. To speed up calculations, voltage-dependent functions in gating variables equations were precomputed and placed into look-up tables.

Additional details on the integration procedure can be found, for example, in [33].

We studied scroll wave dynamics depending on LV wall thickness at the apex and degree of heterogeneity. In our simulations, LV basal thickness was $L=12 \mathrm{~mm}$, while apical thickness was varied to $h=6,12,18 \mathrm{~mm}$. We considered the physiological value of the anisotropy ratio $D_{1} / D_{2}=9$.

Our procedure of postprocessing filament coordinates was thoroughly described in [29].

All calculations were performed on a C program on clusters "URAN" (IMM, Ural Branch of RAS) and "UrFU" of Ural Federal University (Ekaterinburg). The program uses CUDA for GPU parallelization and was compiled with a Nvidia C Compiler "nvcc". Computational nodes have graphical cards Tesla K40m0.

\subsection{Heterogeneity Representation}

The heterogeneity in the ventricular myocardium is a result of changes of balance between inward currents (mainly ICaL) and outward potassium currents. Although in reality these currents are changing in combination, the exact degree of modifiication is not fully quantified. In order to compare effects of the heterogeneities in the same conditions, we decided to study a separate effect of ICaL and one of the most important potassium currents IKs.

We considered LV models with the following types of apicobasal heterogeneity:

1. Apicobasal heterogeneity caused by decrease of ICaL current with $\mathrm{APD}_{\text {base }}<\mathrm{APD}_{\text {apex }}$. We reduced ICaL current at the $\mathrm{LV}$ base to $75 \%, 50 \%$, and $25 \%$ of its original value, which resulted in the gradients of 14, 28, and $38 \mathrm{~ms}$. We denote these cases as ICaL-75, ICaL-50 and ICaL-25.

2. Apicobasal heterogeneity caused by decrease of IKs current with $\mathrm{APD}_{\text {base }}<\mathrm{APD}_{\text {apex }}$. We reduced IKs at the apex to $75 \%, 50 \%$, and $25 \%$ of its original value, which resulted in the APD gradients of 14,34 , and $68 \mathrm{~ms}$. We denote these cases as IKs-75, IKs-50, and IKs-25.

To represent transmural (TM) heterogeneity, we decreased the IK current because IK is the main current affecting APD, and it is responsible for transmural heterogeneity [35]. We followed the work [36] and made models with $\mathrm{APD}_{\text {epi }}<\mathrm{APD}_{\text {endo }}$ by multiplying IKs conductivity by $75 \%$, $50 \%$, and $25 \%$, which resulted in the APD gradients of 12,31 , and $58 \mathrm{~ms}$. We denote these cases as IKs-75-TM, IKs-50-TM, and IKs-25-TM.

The effects of the changes of the currents on AP shape are shown in Figure 1D. Figure 1A shows distribution of APD from apex to base for each type of apicobasal heterogeneity and Figure 1B shows the three types of transmural heterogeneity.

\section{Results}

Below, we present results of our simulations for scroll wave dynamics in ventricles of different geometries and various types of heterogeneity. 
We considered three geometries with the same anisotropy but different in the thickness of the apex $h=6, h=12$, and $h=18 \mathrm{~mm}$. In all these geometries in homogeneous ventricles, the scroll wave drifted to the base and disappeared. We studied the dynamics of the scroll waves with the same initial conditions, but in the presence of the heterogeneity.

We considered two types of regional heterogeneity: apicobasal and transmural.

\subsection{Apicobasal Heterogeneity}

Figure 2A,B show a typical trajectory of the scroll wave drift in the absence of heterogeneity. The scroll was initially located between apex and base, then drifted upwards and disappeared after collision with the boundary at the base. We found that the presence of apicobasal heterogeneity substantially changed the scroll wave dynamics. Figure $2 \mathrm{C}$ shows an example of scroll wave drift from the same initial conditions, but for a small apicobasal heterogeneity of $14 \mathrm{~ms}$, IKs-75. We see that the scroll wave drifts to the apex, stabilizes at $8 \mathrm{~mm}$ from it, and continues its circumferential rotation with a speed of $0.19 \mathrm{~mm} / \mathrm{s}$.

A

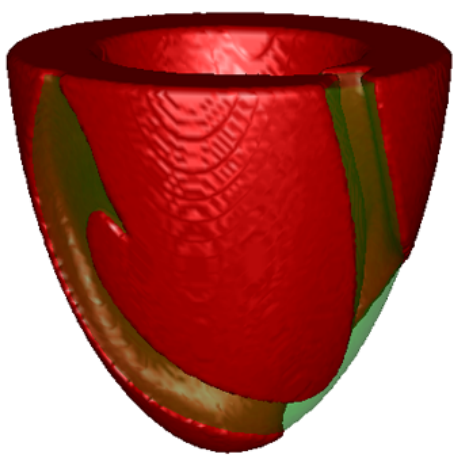

B

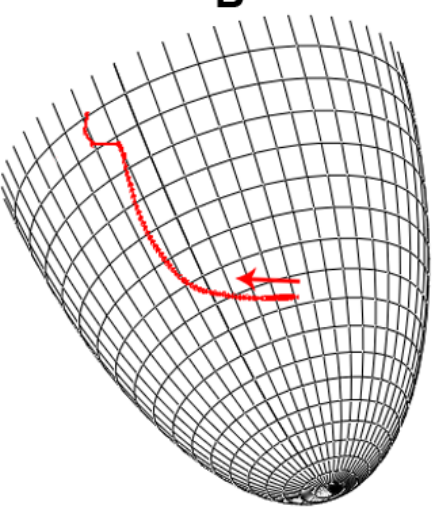

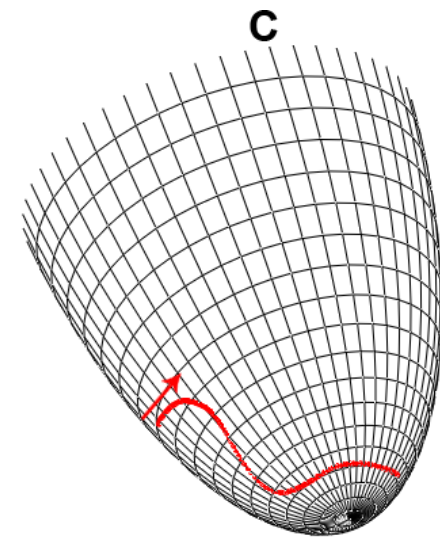

Figure 2. Examples of average filament trajectory. (A) typical view of the scroll wave in the absence of heterogeneity; (B) no heterogeneity; drift to the LV base, where the scroll wave disappeared; (C) apicobasal heterogeneity of $14 \mathrm{~ms}$, IKs-75; drift to the apex, where filament approached a dynamic attractor with vertical coordinate $\Psi=1.24$. The apical thickness $h$ was $6 \mathrm{~mm}$.

We performed 18 computations for six types of the heterogeneity in three LVs of different geometry. The results are presented in Figure 3, where we mark the type of observed dynamics (e.g., annihillaiton at the base and breakup) and in case of stabilisation at attractor we show the coordinates of the attractor, and the drift speed along it.

Our findings were the following. From the 18 cases studied, in only one case (IKs-75 and $h=18 \mathrm{~mm}$ ), we observed the same dynamics as in the homogeneous case: annihilation at the base.

In 12 out of 18 cases, we observed a dramatic change of dynamics: instead of annihilation at the base, the scroll wave stabilized at the apex and then continued moving around it along the circle. One example of such dynamics was already shown in Figure 2C, where the filament stabilized at $8 \mathrm{~mm}$ from the apex and rotated with a speed of $0.19 \mathrm{~mm} / \mathrm{s}$. The apex-base heterogeneity in that case was $14 \mathrm{~ms}$ (the corresponding grey columns in Figure 3A). For the same geometry of $h=6 \mathrm{~mm}$ and a larger heterogeneity (IKs-25, $D R_{A B}=68 \mathrm{~ms}$ ), the scroll wave filament stabilized also at approximately $8 \mathrm{~mm}$ from the apex $(\psi=1.24)$ and was moving along the circle with speed $2.27 \mathrm{~mm} / \mathrm{s}$. The speed of the motion along the attractor increased with the increase in the heterogeneity. Note that the location of attractor in most of the cases did not depend on the degree of heterogeneity. However, for $h=18 \mathrm{~mm}$, we saw that the location of the attractor for ICaL-50 was closer to the base than for ICaL-75. 
A

$(\mathrm{h}=6$, thin apex)
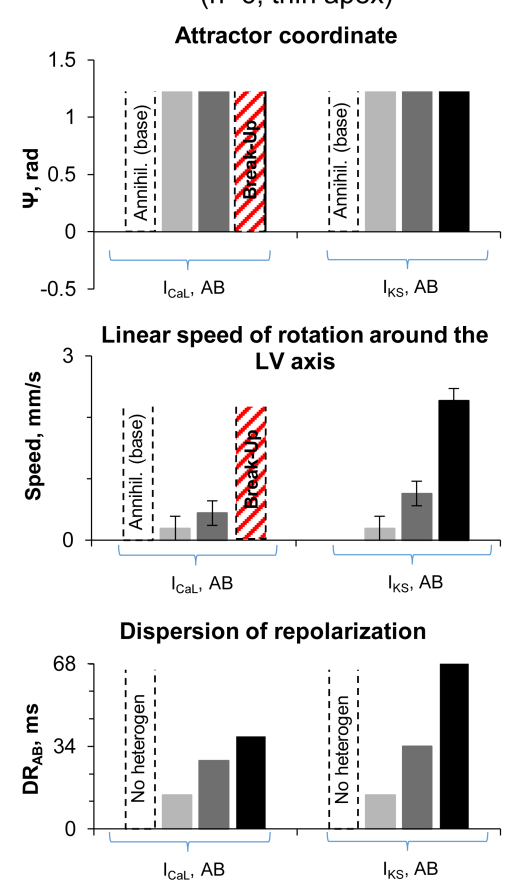

B

$(\mathrm{h}=12$, medium apex)

Attractor coordinate
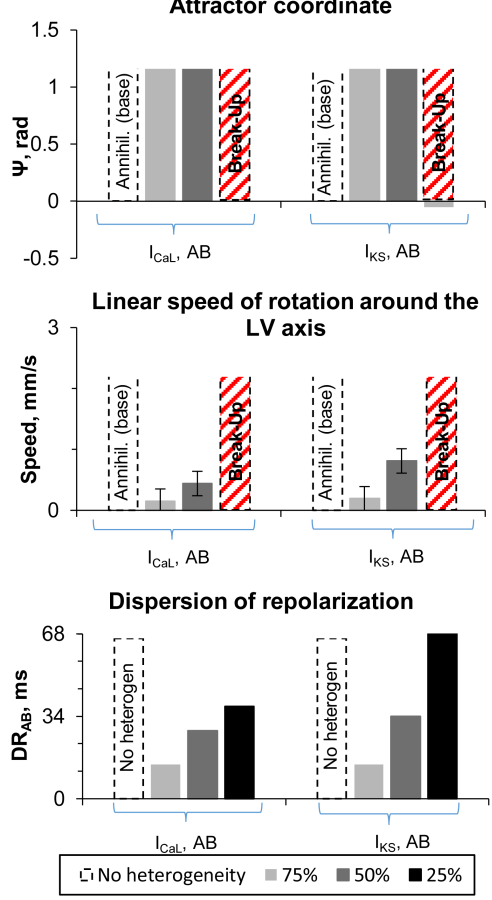

C

$(\mathrm{h}=18$, thick apex) Attractor coordinate
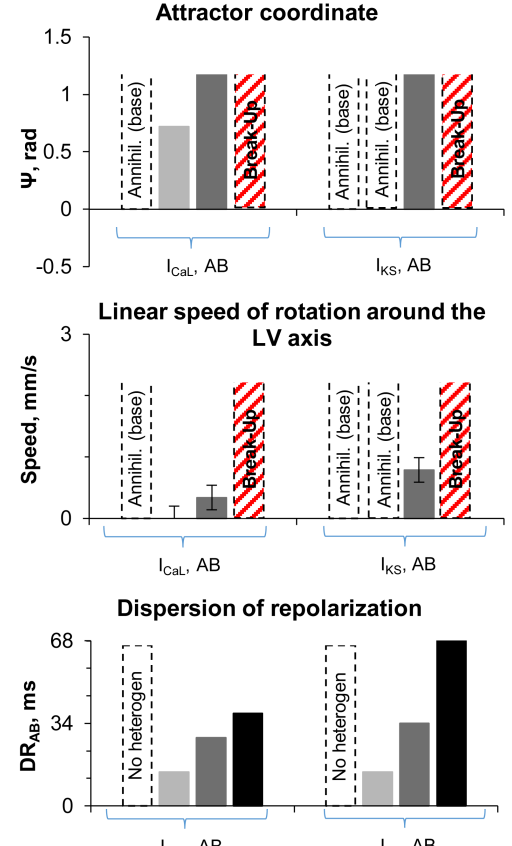

Figure 3. Results of numerical experiments on LV models with apicobasal heterogeneity. (A-C) LV models with apical thickness 6, 12, and $18 \mathrm{~mm}$, respectively. Top row: Attractor coordinate. Dotted bars mean that scroll wave disappeared at the LV base. Red dotted bars show the cases with wave break up. Middle row: Linear speed of rotation around the LV axis. Bottom row: Apicobasal dispersion of repolarization $\left(D R_{A B}\right)$ for points with coordinates $\Psi=0$ and $\Psi=\pi / 2$ (see Figure 1).

In the five other cases, all with large heterogeneity, we observed a more complex dynamics. Here, the initial scroll wave generated waves with a high frequency. Such waves were propagating toward the apex where the APD and refractory period were the longest and tissue was not able to recover. As a result, we observed formation of the wave breaks. Figure 4 shows an example of such process. Here, the scroll wave, whose tip is located at the other side of the LV, generates two wavebreaks (Figure 4B) which evolve to a complex spatiotemporal pattern (Figure 4C). This occurred in cases IKs-25 and ICaL-25.

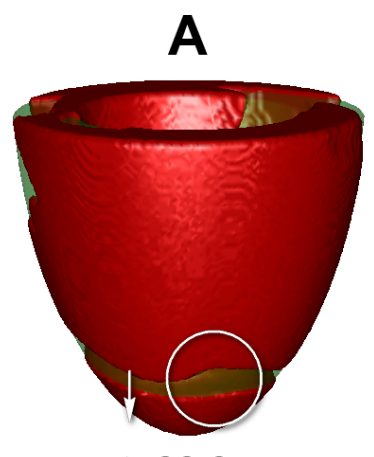

$\mathrm{t}=63.3 \mathrm{~s}$

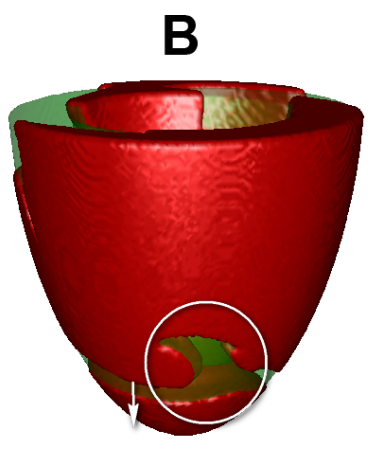

$\mathrm{t}=63.7 \mathrm{~s}$

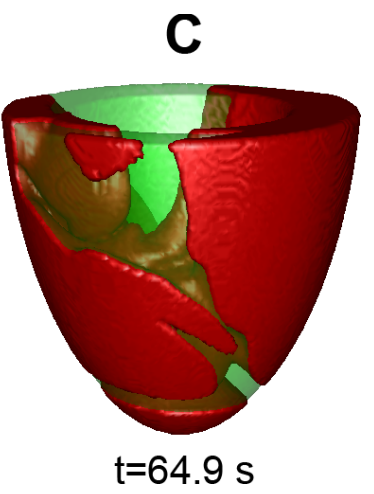

$\mathrm{t}=64.9 \mathrm{~s}$

Figure 4. Example of wave break on the LV with apical thickness $6 \mathrm{~mm}$, apicobasal heterogeneity ICaL-25. (A-C) show wave patterns at $t=63.3 \mathrm{~s} ; \mathrm{t}=63.7 \mathrm{~s} . ; \mathrm{t}=64.9 \mathrm{~s}$.

Interestingly, long filaments with both ends at the epicardial surface sometimes emerged (Figure 5). Such filament may have a complex behavior which can lead to annihilation of the pattern or formation of new filaments. However, they never stabilized to attractors as in the previous 12 cases. 

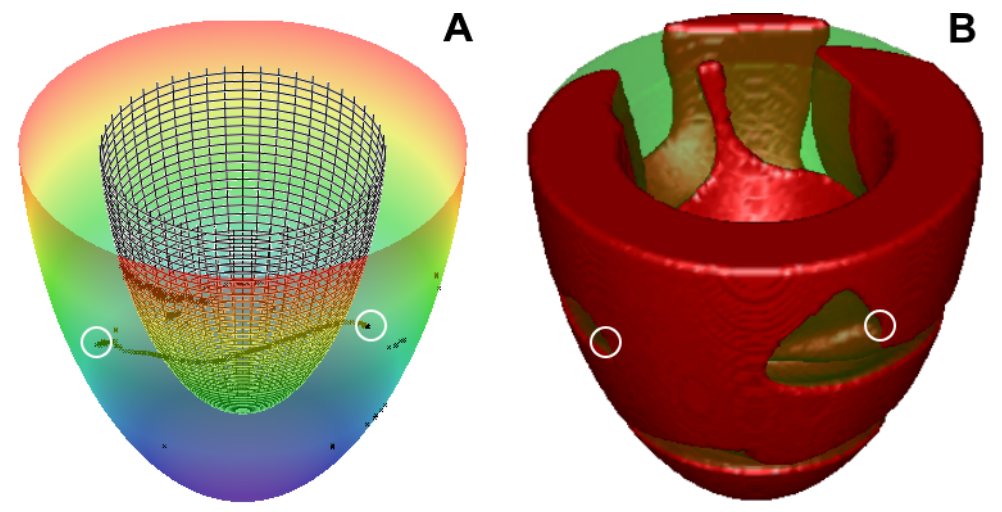

Figure 5. Scroll wave in the LV can have a filament with both ends at the epicardium. (A) the filament shape at $\mathrm{t}=25.4 \mathrm{~s}$ for the LV with apical thickness $\mathrm{h}=6 \mathrm{~mm}$, apicobasal heterogeneity, IKs-25. We see that the filament is located between the LV epicardium (colorful surface) and endocardium (meshy surface); (B) the scroll wave at the same time (red area shows excited cells). Ends of the filament are marked with white circles.

Overall, we can say that, while a moderate increase in heterogeneity resulted in stabilization of filament towards the region of longer APD, a larger degree of heterogeneity resulted in the formation of additional wavebreaks and complex spatio-temporal patterns.

\subsection{Transmural Heterogeneity}

To investigate the effect of transmural heterogeneity, we performed nine simulations: three LVs with different geometry with three degrees of heterogeneity, where we changed IKs. This is because IK is the main ionic current responsible for the transmural heterogeneity. The results of these simulations are shown in Figure 6.

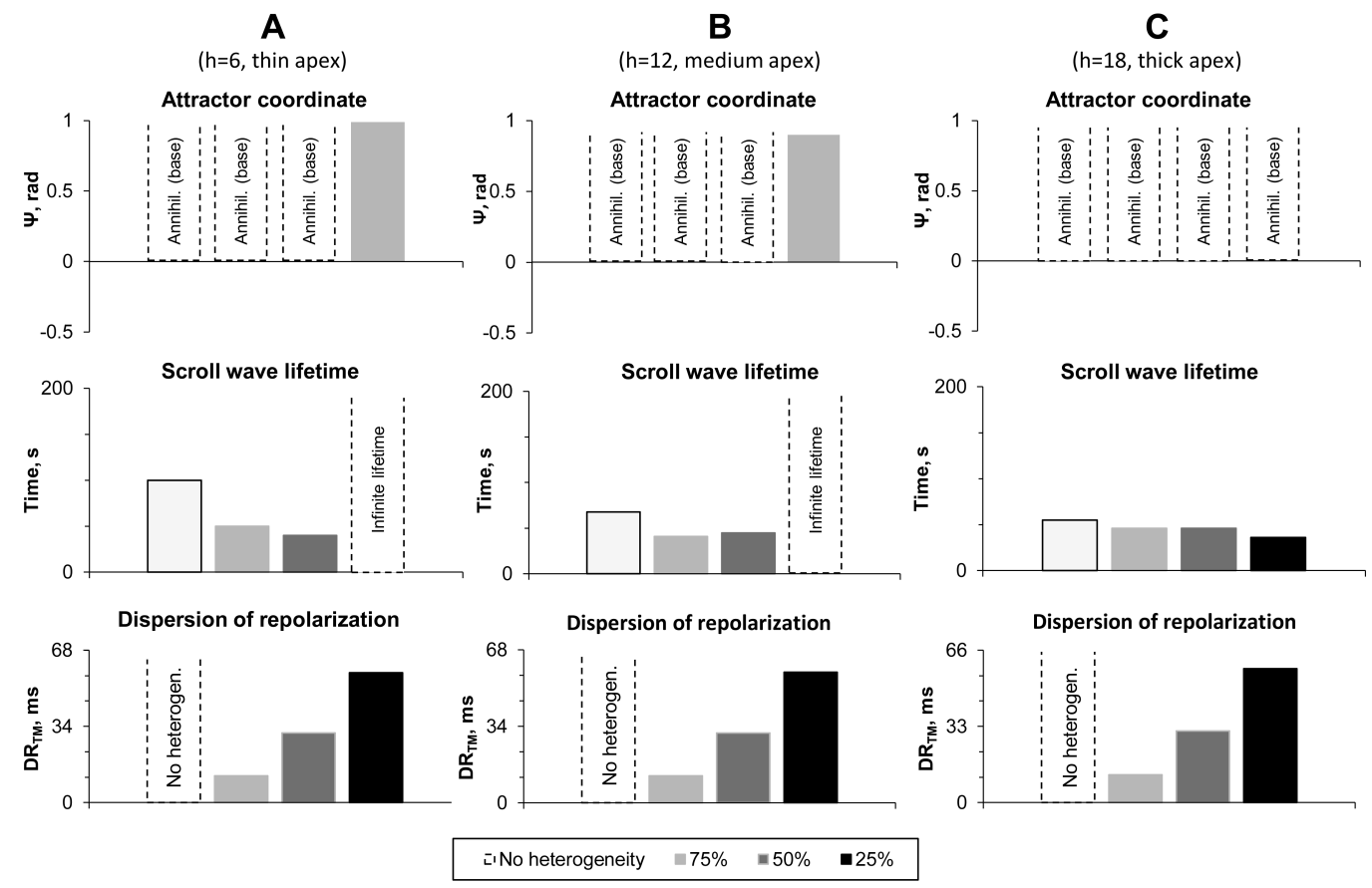

Figure 6. Results of numerical experiments on LV models with transmural heterogeneity. (A-C): LV models with apical thickness 6, 12, and $18 \mathrm{~mm}$, respectively. Top row: Attractor coordinates. Dotted bars mean that scroll wave disappeared at the LV base. Middle row: Scroll wave lifetime (200 means it survived until the end of the simulation). Bottom row: Transmural dispersion of repolarization $\left(D R_{T M}\right)$ for points with coordinates $\gamma=0.1$ and $\gamma=0.9$ (see Figure $1 C$ ). 
In seven out of nine cases, we observed the same type of dynamics as in the absence of the heterogeneity. The scroll wave drifted to base and annihilated there. We saw, however, that the lifetime of the scroll wave decreased with increase in heterogeneity. In two cases (IKs-25-TM; apex thickness 6 and $12 \mathrm{~mm}$ ), the scroll wave stabilized at the apex and either rotated with a velocity of $0.19 \mathrm{~mm} / \mathrm{s}$ $(h=6 \mathrm{~mm})$ or did not move at all $(h=12 \mathrm{~mm})$.

\section{Discussion}

In this paper, we study the effects of heterogeneity on scroll wave dynamics in a model of the left ventricles of the human heart. We used a detailed ionic model for human ventricular cell and analytical representation of the geometry and anisotropy. We considered two types of heterogeneity: apicobasal and transmural, and we represent them by changing ICaL and IK currents.

The apicobasal heterogeneity was found to change the dynamics of the scroll wave. Instead of annihilation at the base, the scroll wave in most of the cases stabilized at the apex. This result can be explained by the following known dynamics of the spiral wave in cardiac tissue. In [20,21], it was shown that spiral waves drift to the region of longer APD. Because in our case APD was longest at the apex, we can assume that stabilization at the apex is a result of such dynamics. Note that for $h=6 \mathrm{~mm}$ and $12 \mathrm{~mm}$ the location of the attractor did not change with the degree of the heterogeneity. However, for $h=18 \mathrm{~mm}$, we had a gradual change of the location of the attractor $\Psi=0.72$ for ICaL-75, while $\Psi=1.2$ for ICaL-50. In addition, for $h=18 \mathrm{~mm}$ and IKs-75, the scroll wave still drifted to the base and annihilated there. The mechanisms of the observed phenomenon can be the following. In case $h=18 \mathrm{~mm}$, the apex is substantially thicker than the base. Because in the absence of heterogeneities the scroll wave drifts toward the region of minimal thickness, such gradient attempts to push the scroll wave towards the base. On the other hand, the apicobasal heterogeneity thrusts the scroll wave toward the apex. As a result of interaction of these opposite processes, we observe a stabilization of the scroll closer to the apex for larger heterogeneity in ICaL. In addition, the small heterogeneity IKs-75 was not sufficient to stabilize the scroll and it annihilated at the base.

Another important type of the dynamics is a breakup. Basically in all cases of large heterogeneity, some form of breakup was observed. These results can also be explained in the following way. The formation of wavebreak at the regions with a longer refractory period (APD) is one of the most classical mechanisms of generation of spiral waves in 2D [37]. As in our case, the breakup occurred via formation of wavebreak at the apex, where the APD is longest, we think that the mechanism [37] is also responsible for the onset of breakup in our case.

For the transmural heterogeneity, we did not observe substantial effects on the filament dynamics. It did not change the location of the attractor: in almost all cases as in the control case, the scroll wave drifted towards the base and annihilated there. We saw a slight decrease in the life time of the scroll wave, but the effect was small and not significant. In a few cases of largest heterogeneity, we saw stabilization at the apex. Unfortunately, the mechanisms of such behaviour are not clear. In [22,23], it was shown that transmural heterogeneity affects the period of rotation of a scroll wave, and in extreme cases can cause its breakup. However, studies there were perfomed in a simplified model of cardiac tissue. In [38], a sproing (elongation) of the filament was also observed in a simplified model of cardiac tissue. However, we did not observe such phenomena and found effects that require additional study.

It should be noted that filaments have complex dynamics; however, not all complexity of the dynamics comes from the heterogeneity. As pointed out by Papadimitriou [39], the complexity does not necessarily require heterogeneity and that is definitely true for filaments. For example, even in completely homogeneous cardiac tissue, for certain parameter values, one can have a breakup of filaments due to negative filament tension [40], or dynamic instabilities [41]. In addition, anisotropy by itself can produce complex filament shapes [42]. It would be interesting to further characterize complexity of filament dynamics in the heart in a wider range of parameters and study if heterogeneity will be a determinant for their dynamics as it was in our study. 
In a previous paper [26], we studied scroll wave drift on the Aliev-Panfilov model and symmetrical LV model with isotropy and anisotropy 9:1 in homogeneous and heterogeneous myocardium. In that paper, we also found a tendency of scroll wave to move towards the apex and an increase of the rotation speed of waves with an increase in the heterogeneity. The drift speed in the AP model was smaller than one found in this paper, which is a result of using of a more accurate model for cardiac excitation.

Overall, these results show that the scroll wave drifts and stabilizes at the apex of the left ventricle in the presence of moderate apicobasal heterogeneity. Thus, independently of their initial location, the scroll waves are after some transient processes more likely to be observed at the apex of the heart. Therefore, the apex may be a preferable location of the scroll wave. Identification of the location of a source of cardiac arrhythmias is an important question in practical cardiology. One of the most effective ways to treat cardiac arrhythmia is a cardiac ablation procedure, during which flexible catheters are passed into the heart through a blood vessel. Once located near the target region, the catheter is activated to locally destroy or modify the arrhythmia substrate by delivering radio-frequency energy or extreme cold. Thus, locating the arrhythmia source is a problem of great importance. In this paper we show that the source of cardiac arrhythmia is more likely to be located at the apex of the heart. Hence, the modification of the apex by some sort of ablation, which makes rotation of scroll wave impossible, can eliminate the cardiac arrhythmia. Recently, a non-invasive method of ablation was proposed, which enables modifying the tissue not only on the surface but also inside the myocardial wall by noninvasive delivery of radiation with stereotactic body radiation [43]. For such methodology, the location of the arrhythmia source is again crucial. Note, however, that ablation in 3D ventricle to eliminate a scroll wave is a non-trivial issue and needs to be further studied, e.g., using a modeling methodology applied in our paper.

A limitation of our approach is that we considered only spiral waves with one chirality. The chirality may substantially affect the dynamics of scroll waves [29]. In this paper, we chose that particular case for counterclockwise rotation as without heterogeneity we had always one regime (annihilation at the base). It was thus straightforward to study the effect of heterogeneity in that situation. The effect of chirality in the presence of heterogeneity should be investigated in a subsequent study.

We studied transmural and apicobasal heterogeneity separately. It would be also interesting to study their combined effect on scroll wave dynamics.

In this paper, we performed our studies only in a limited parameter range. This is because such modeling is extremely challenging, as the studied effects can be observed only in anatomical models of the heart on a long time scale (of the order of minutes) while typical simulations in anatomical models of the heart are normally performed for a few seconds (see e.g., [44]). It would be interesting in the future to extend studies for a larger parameter range and also model direct clinical interventions which can stop arrhythmias caused by scroll waves, such as catheter ablation.

\section{Conclusions}

We performed a study of filament dynamics in the left ventricle of the heart using a state-of-art ionic model for cardiac tissue and combining all the most important factors which can be responsible for the filament drift: the shape of the ventricle, variation in thickness, anisotropy, and heterogeneity. Under those conditions, we find that, in spite of the importance of all factors, the apicobasal heterogeneity was the most important in determining the final position of the filament. For moderate heterogeneity, the filament was stabilized at the apex of the ventricle, while for a large heterogeneity a break-up into a complex spatio-temporal pattern was observed. The transmural heterogeneity did not substantially affect the filament dynamics. As predicting of location of the arrhythmia sources is important for clinical interventions, our results can help in developing clinical procedures to remove arrhythmias organized by filaments. 
Author Contributions: Conceptualization, A.V.P. and O.S.; methodology, A.V.P. and S.P.; software S.P., H.D., and P.K.; formal analysis, S.P.; investigation, P.K.; writing-original draft preparation, P.K. and S.P.; writing-review and editing, P.K., S.P., A.V.P., H.D., and O.S.; supervision, A.V.P. and O.S. All authors have read and agreed to the published version of the manuscript.

Funding: P.K., S.P., O.S., and A.V.P. were funded by the Russian Science Foundation (project 14-35-00005). A.V.P., P.K., and O.S. were funded by the Russian Foundation for Basic Research (\#18-29-13008). A.V.P. and O.S. were funded by RF Government Act \#211 of 16 March 2013 (agreement 02.A03.21.0006). P.K. and O.S. work was carried out within the framework of the IIF UrB RAS theme No. AAAA-A18-118020590031-8. A.V.P. and H.D. were partially funded by BOF Ghent University.

Acknowledgments: Simulations were performed at the supercomputer Uran of Institute of Mathematics and Mechanics (Ekaterinburg, Russia) and at the supercomputer of Ural Federal University (Ekaterinburg, Russia).

Conflicts of Interest: The authors declare no conflict of interest.

\section{References}

1. Winfree, A.T.; Strogatz, S.H. Organizing centres for three-dimensional chemical waves. Nature 1984, 311, 611-615. [CrossRef] [PubMed]

2. Mehra, R. Global public health problem of sudden cardiac death. J. Electrocardiol. 2007, 40, S118-S122. [CrossRef] [PubMed]

3. Gray, R.A.; Jalife, J.; Panfilov, A.; Baxter, W.T.; Cabo, C.; Davidenko, J.M.; Pertsov, A.M. Nonstationary Vortexlike Reentrant Activity as a Mechanism of Polymorphic Ventricular Tachycardia in the Isolated Rabbit Heart. Circulation 1995, 91, 2454-2469. [CrossRef] [PubMed]

4. Katz, A.M. Physiology of the Heart; Lippincott Williams \& Wilkins: Philadelphia, PA, USA, 2010.

5. Panfilov, A.; Rudenko, A.; Krinsky, V. Scroll rings in three-dimensional active medium with two component diffusion. Biofizika 1986, 31, 850-854.

6. Panfilov, A.; Aliev, R.; Mushinsky, A. An integral invariant for scroll rings in a reaction-diffusion system. Phys. D Nonlinear Phenom. 1989, 36, 181-188. [CrossRef]

7. Pravdin, S.; Dierckx, H.; Markhasin, V.S.; Panfilov, A.V. Drift of Scroll Wave Filaments in an Anisotropic Model of the Left Ventricle of the Human Heart. BioMed Res. Int. 2015, 2015. [CrossRef]

8. Rogers, J.M.; McCulloch, A.D. Nonuniform muscle fiber orientation causes spiral wave drift in a finite element model of cardiac action potential propagation. J. Cardiovasc. Electrophysiol. 1994, 5, 496-509. [CrossRef]

9. Dierckx, H.; Bernus, O.; Verschelde, H. A geometric theory for scroll wave filaments in anisotropic excitable media. Phys. D Nonlinear Phenom. 2009, 238, 941-950. [CrossRef]

10. Nabauer, M.; Beuckelmann, D.J.; Uberfuhr, P.; Steinbeck, G. Regional Differences in Current Density and Rate-Dependent Properties of the Transient Outward Current in Subepicardial and Subendocardial Myocytes of Human Left Ventricle. Circulation 1996, 93, 168-177. [CrossRef]

11. Glukhov, A.V.; Fedorov, V.V.; Lou, Q.; Ravikumar, V.K.; Kalish, P.W.; Schuessler, R.B.; Moazami, N.; Efimov, I.R. Transmural Dispersion of Repolarization in Failing and Nonfailing Human Ventricle. Circ. Res. 2010, 106, 981-991. [CrossRef]

12. Opthof, T.; Janse, M.J.; Meijborg, V.M.; Cinca, J.; Rosen, M.R.; Coronel, R. Dispersion in ventricular repolarization in the human, canine and porcine heart. Prog. Biophys. Mol. Biol. 2016, 120, 222-235. [CrossRef] [PubMed]

13. Franz, M.R.; Bargheer, K.; Rafflenbeul, W.; Haverich, A.; Lichtlen, P.R. Monophasic action potential mapping in human subjects with normal electrocardiograms: Direct evidence for the genesis of the T wave. Circulation 1987, 75, 379-386. [CrossRef] [PubMed]

14. Janse, M.J.; Coronel, R.; Opthof, T.; Sosunov, E.A.; Anyukhovsky, E.P.; Rosen, M.R. Repolarization gradients in the intact heart: Transmural or apico-basal? Prog. Biophys. Mol. Biol. 2012, 109, 6-15. [CrossRef]

15. Boukens, B.J.; Sulkin, M.S.; Gloschat, C.R.; Ng, F.S.; Vigmond, E.J.; Efimov, I.R. Transmural APD gradient synchronizes repolarization in the human left ventricular wall. Cardiovasc. Res. 2015, 108, 188-196. [CrossRef] [PubMed]

16. Szentadrassy, N.; Banyasz, T.; Biro, T.; Szabo, G.; Toth, B.I.; Magyar, J.; Lazar, J.; Varro, A.; Kovacs, L.; Nanasi, P.P. Apico-basal inhomogeneity in distribution of ion channels in canine and human ventricular myocardium. Cardiovasc. Res. 2005, 65, 851-860. [CrossRef] 
17. Solovyova, O.; Katsnelson, L.; Konovalov, P.; Kursanov, A.; Vikulova, N.; Kohl, P.; Markhasin, V. The cardiac muscle duplex as a method to study myocardial heterogeneity. Prog. Biophys. Mol. Biol. 2014, 115, 115-128. [CrossRef]

18. Antzelevitch, C. Heterogeneity and cardiac arrhythmias: An overview. Heart Rhythm 2007, 4, $964-972$. [CrossRef]

19. Keldermann, R.H.; ten Tusscher, K.H.; Nash, M.P.; Hren, R.; Taggart, P.; Panfilov, A.V. Effect of heterogeneous APD restitution on VF organization in a model of the human ventricles. Am. J. Physiol.-Heart Circ. Physiol. 2008, 294, H764-H774. [CrossRef]

20. Rudenko, A.; Panfilov, A. Drift and interaction of vortices in two-dimensional heterogeneous active medium. Stud. Biophys. 1983, 98, 183-188.

21. Ten Tusscher, K.; Panfilov, A.V. Reentry in heterogeneous cardiac tissue described by the Luo-Rudy ventricular action potential model. Am. J. Physiol.-Heart Circ. Physiol. 2003, 284, H542-H548. [CrossRef]

22. Panfilov, A.V.; Rudenko, A.N.; Pertsov, A.M. Twisted scroll waves in three- dimensional active media. Dokl. Akad. Nauk SSSR 1984, 279, 1000-1002.

23. Panfilov, A.V.; Keener, J.P. Twisted scroll waves in heterogeneous excitable media. Int. J. Bifurc. Chaos 1993, 3, 445-450. [CrossRef]

24. Pravdin, S.F.; Berdyshev, V.I.; Panfilov, A.V.; Katsnelson, L.B.; Solovyova, O.; Markhasin, V.S. Mathematical model of the anatomy and fibre orientation field of the left ventricle of the heart. Biomed. Eng. Online 2013, 12, 54. [CrossRef]

25. Pravdin, S.F.; Dierckx, H.; Katsnelson, L.B.; Solovyova, O.; Markhasin, V.S.; Panfilov, A.V. Electrical wave propagation in an anisotropic model of the left ventricle based on analytical description of cardiac architecture. PLoS ONE 2014, 9. [CrossRef]

26. Konovalov, P.V.; Pravdin, S.F.; Solovyova, O.E.; Panfilov, A.V. Scroll Wave dynamics in a model of the heterogeneous heart. JETP Lett. 2016, 104, 821-821. [CrossRef]

27. Aliev, R.R.; Panfilov, A.V. A simple two-variable model of cardiac excitation. Chaos Solitons Fractals 1996, 7, 293-301. [CrossRef]

28. Panfilov, A. Three-dimensional organization of electrical turbulence in the heart. Phys. Rev. E 1999, 59, R6251-R6254. [CrossRef]

29. Pravdin, S.F.; Dierckx, H.; Panfilov, A.V. Drift of scroll waves in a generic axisymmetric model of the cardiac left ventricle. Chaos Solitons Fractals 2019, 120, 222-233. [CrossRef]

30. Streeter, D., Jr. The Heart. In Handbook of physiology; Section 2; American Physiological Society: Bethesda, MD, USA, 1979; Chapter Gross Morphology and Fiber Geometry of the Heart; Volume I, pp. 61-112.

31. Panfilov, A.; Holden, A. Computer simulation of re-entry sources in myocardium in two and three dimensions. J. Theor. Biol. 1993, 161, 271-285. [CrossRef]

32. ten Tusscher, K.; Panfilov, A.V. Alternans and spiral breakup in a human ventricular tissue model. Am. J. Physiol.-Heart Circ. Physiol. 2006, 291, H1088-H1100. [CrossRef]

33. Kazbanov, I.V.; Clayton, R.H.; Nash, M.P.; Bradley, C.P.; Paterson, D.J.; Hayward, M.P.; Taggart, P.; Panfilov, A.V. Effect of Global Cardiac Ischemia on Human Ventricular Fibrillation: Insights from a Multi-scale Mechanistic Model of the Human Heart. PLoS Comput. Biol. 2014, 10, e1003891. [CrossRef] [PubMed]

34. Rush, S.; Larsen, H. A practical algorithm for solving dynamic membrane equations. IEEE Trans. Biomed. Eng. 1978, 4, 389-392. [CrossRef] [PubMed]

35. Pereon, Y.; Demolombe, S.; Baro, I.; Drouin, E.; Charpentier, F.; Escande, D. Differential expression of KvLQT1 isoforms across the human ventricular wall. Am. J. Physiol.-Heart Circ. Physiol. 2000, 278, H1908-H1915. [CrossRef]

36. ten Tusscher, K.; Noble, D.; Noble, P.J.; Panfilov, A.V. A model for human ventricular tissue. Am. J. Physiol.-Heart Circ. Physiol. 2004, 286, H1573-H1589. [CrossRef] [PubMed]

37. Krinskii, V. Spread of excitation in an inhomogeneous medium (state similar to cardiac fibrillation). Biophysics-USSR 1966, 11, 676-683.

38. Henze, C.; Lugosi, E.; Winfree, A. Helical organizing centers in excitable media. Can. J. Phys. 1990, 68, 683-710. [CrossRef]

39. Papadimitriou, F. Geo-mathematical modeling of spatial-ecological complex systems: An evaluation. Geogr. Environ. Sustain. 2010, 3, 67-80. [CrossRef] 
40. Biktashev, V.; Holden, A.; Zhang, H. Tension of organizing filaments of scroll waves. Philos. Trans. R. Soc. London. Ser. A Phys. Eng. Sci. 1994, 347, 611-630.

41. Panfilov, A.V.; Hogeweg, P. Mechanisms of cardiac fibrillation. Science 1995, 270, 1223-1224.

42. Verschelde, H.; Dierckx, H.; Bernus, O. Covariant stringlike dynamics of scroll wave filaments in anisotropic cardiac tissue. Phys. Rev. Lett. 2007, 99, 168104. [CrossRef]

43. Cuculich, P.S.; Schill, M.R.; Kashani, R.; Mutic, S.; Lang, A.; Cooper, D.; Faddis, M.; Gleva, M.; Noheria, A.; Smith, T.W.; et al. Noninvasive cardiac radiation for ablation of ventricular tachycardia. N. Engl. J. Med. 2017, 377, 2325-2336. [CrossRef] [PubMed]

44. Arevalo, H.J.; Vadakkumpadan, F.; Guallar, E.; Jebb, A.; Malamas, P.; Wu, K.C.; Trayanova, N.A. Arrhythmia risk stratification of patients after myocardial infarction using personalized heart models. Nat. Commun. 2016, 7, 11437. [CrossRef] [PubMed]

(C) 2020 by the authors. Licensee MDPI, Basel, Switzerland. This article is an open access article distributed under the terms and conditions of the Creative Commons Attribution (CC BY) license (http://creativecommons.org/licenses/by/4.0/). 